Supplement of Atmos. Chem. Phys. Discuss., 14, 31039-31090, 2014

http://www.atmos-chem-phys-discuss.net/14/31039/2014/

doi:10.5194/acpd-14-31039-2014-supplement

(C) Author(s) 2014. CC Attribution 3.0 License.

(c) (1)

\title{
Evaluation of a regional chemistry transport model using a newly developed regional $\mathrm{OMI} \mathrm{NO}_{2}$ retrieval
}

G. Kuhlmann et al.

Correspondence to: G. Kuhlmann (gerrit.kuhlmann@gmail.com) and Y. F. Lam (yunflam@cityu.edu.hk) 
Table 1: Evaluation of WRF with meteorological observations in Hong Kong.

\begin{tabular}{lrrrrrr}
\hline Parameter & IOA & $\mathrm{r}$ & $\mathrm{MB}$ & $\mathrm{NMB}^{a}$ & $\mathrm{RMSE}$ & $\mathrm{CV}^{a}$ \\
\hline Hong Kong Observatory & (HKO) & & & \\
$T\left({ }^{\circ} \mathrm{C}\right)$ & 0.97 & 0.96 & -0.2 & -0.8 & 1.7 & 8.0 \\
$q(\mathrm{~g} / \mathrm{kg})$ & 0.93 & 0.94 & -1.7 & -14.1 & 2.2 & 18.2 \\
$p(\mathrm{hPa})$ & 0.99 & 0.98 & -0.0 & -0.0 & 0.9 & 0.1 \\
$v(\mathrm{~m} / \mathrm{s})$ & 0.57 & 0.28 & +0.5 & +23.2 & 1.5 & 71.4 \\
\hline Hong Kong International & Airport $(\mathrm{HKIA})$ \\
$T\left({ }^{\circ} \mathrm{C}\right)$ & 0.98 & 0.97 & -0.2 & -0.7 & 1.3 & 6.1 \\
$q(\mathrm{~g} / \mathrm{kg})$ & 0.97 & 0.95 & -0.3 & -2.3 & 1.4 & 12.8 \\
$p(\mathrm{hPa})$ & 0.99 & 0.98 & +0.1 & +0.0 & 0.9 & 0.1 \\
$v(\mathrm{~m} / \mathrm{s})$ & 0.68 & 0.47 & -0.4 & -10.4 & 1.6 & 46.0 \\
\hline Waglan Island $(\mathrm{WGL})$ & \multicolumn{5}{l}{} \\
$T\left({ }^{\circ} \mathrm{C}\right)$ & 0.94 & 0.94 & +1.2 & +5.7 & 2.0 & 9.5 \\
$q(\mathrm{~g} / \mathrm{kg})$ & 0.88 & 0.95 & +2.5 & +21.7 & 2.8 & 24.2 \\
$p(\mathrm{hPa})$ & 0.99 & 0.98 & +0.4 & +0.0 & 1.0 & 0.1 \\
$v(\mathrm{~m} / \mathrm{s})$ & 0.84 & 0.72 & +0.0 & +0.7 & 2.1 & 33.0 \\
\hline$T:$ temperature at & $2 m$ above ground level (agl), $q:$ water vapour \\
mixing ratio $2 m$ agl, $p:$ sea level pressure, $v:$ wind speed (at \\
station height), ${ }^{a}$ unit percent \\
\hline
\end{tabular}

Table 2: Evaluation of CMAQ $\mathrm{NO}_{2}$ mixing ratios with the PRD RAQM network.

\begin{tabular}{|c|c|c|c|c|c|c|}
\hline Station name & IOA & $\mathrm{r}$ & MB & NMB & RMSE & $\mathrm{CV}$ \\
\hline Chengzhong & 0.47 & +0.22 & -13.1 & -50 & 21.2 & 82 \\
\hline Donghu & 0.44 & +0.21 & +18.7 & +80 & 27.6 & 118 \\
\hline Haogang & 0.59 & +0.37 & -9.2 & -30 & 20.4 & 65 \\
\hline Huijingcheng & 0.60 & +0.49 & -18.7 & -40 & 28.9 & 62 \\
\hline Jinguowan & 0.42 & +0.19 & -2.6 & -46 & 5.9 & 105 \\
\hline Liyuan & 0.58 & +0.37 & +14.4 & +41 & 25.0 & 70 \\
\hline Luhu Park & 0.71 & +0.60 & -14.0 & -38 & 23.6 & 64 \\
\hline Shunde D. & 0.47 & +0.22 & -23.7 & -61 & 31.8 & 81 \\
\hline Tangjia & 0.50 & +0.17 & -13.4 & -41 & 27.6 & 85 \\
\hline Tap Mun & 0.29 & -0.09 & -2.3 & -26 & 10.5 & 118 \\
\hline Tianhu & 0.46 & +0.25 & -4.2 & -60 & 7.6 & 110 \\
\hline Tsuen Wan & 0.75 & +0.58 & -1.4 & -3 & 17.9 & 41 \\
\hline Tung Chung & 0.63 & +0.45 & -10.9 & -30 & 22.0 & 61 \\
\hline Wanqingsha & 0.43 & +0.07 & -13.8 & -39 & 29.3 & 82 \\
\hline Xiapu & 0.48 & +0.42 & -12.8 & -67 & 19.1 & 100 \\
\hline Zimaling $\mathrm{P}$. & 0.57 & +0.32 & -10.2 & -24 & 23.3 & 56 \\
\hline HK \& SZ & 0.56 & +0.33 & -0.0 & -5 & 18.9 & 73 \\
\hline FS \& GZ & 0.55 & +0.34 & -17.6 & -44 & 28.4 & 72 \\
\hline all stations & 0.52 & +0.30 & -7.3 & -27 & 21.4 & 81 \\
\hline
\end{tabular}

